\title{
Finger Vein Recognition using Straight Line Approximation based on Ensemble Learning
}

\author{
Roza Waleed Ali ${ }^{1}$, Junaidah Mohamed Kassim², Siti Norul Huda Sheikh Abdullah ${ }^{3}$ \\ Faculty of Information Science and Technology \\ Universiti Kebangsaan Malaysia \\ Bangi, Selangor, Malaysia
}

\begin{abstract}
Human identity recognition and protection of information security are current global concerns in this age of increasing information growth. Biometrics approach of defining identity is considered as one of the highly potential approaches due to its internal feature that is difficult to be artificially recreated, stolen and/or forgotten. The new recognition system based on finger vein is a unique method depending on physiological traits and parameters of the vein patterns for the human. Published works on finger vein identification have hitherto ignored the power of aggregating different types of features and classifiers in improving the performance of the biometric recognition system. In this paper, we developed a novel feature approach named as straight line approximator (SLA) for extending the feature space of vein pattern using a public data set SDUMLA-HMT comprising about 3,816 images of finger vein for 160 persons. Furthermore, we applied a set of extreme learning machine (ELM) and support vector machine (SVM) classifier in different kernels. Then, we used the combination rules to improve the performance of the system. The experiment result of the proposed method achieved an accuracy of $87 \%$ using (DS and GWAR) rules at rank 1, while the accuracy of DS rule $93 \%$ and GWAR rule $92 \%$ at rank 5.
\end{abstract}

Keywords-Finger vein recognition; SLA; ELM; SVM; HOG; straight line approximate

\section{INTRODUCTION}

In this rising age in information security, the main social problem at hand is how to solve human identity recognition and protect information security. The conventional identity recognition contains two types of methods. The first is content-based, which are: a password, code [1] and so on, and the second method is a possessing based, which are: a smart card, license, and others. The technologies based on biometric have been made possible by explosive advances in computing power and the near general interconnection of computers around the world. Therefore, these are used in the variety of ranges in national applications, like physical access control, banking security, customs and immigration, information system security, digital forensics [2] voters and national ID systems[3]. Also, in applications such as mobile security, network authentication, time tracking and attendance of employees, viz à viz, credit card authentication is all involved in the use of biometrics.

Biometric can be defined as the science through which individual identification can be verified, established and recognized automatically based on behavioral traits [4] (speech, handwriting, and signature) or physiological features
[5][6][7] (gait, hand vein, fingerprint, finger vein, and face). The law enforcement agencies were the ones who first used biometric systems in the 1970s to investigate criminals through the using of fingerprint recognition [8]. However, the current development in biometric technologies and increasing threats have formed a risk for the information security, which has caused the increasing growth of the application of biometric systems in both access control domains [9], such as physical and logical. There is now an encouragement for these kinds of biometric systems because the biometric capturing machines cost less than other machines [10]. Researchers [11] presented the comparison of major biometrics techniques by defining the advantages and disadvantages as shown in Table 1.

TABLE I. COMPARISON OF MAJOR BIOMETRICS TECHNIQUES [11]

\begin{tabular}{|l|l|l|l|l|l|}
\hline Biometrics & Accuracy & Size & Cost & $\begin{array}{l}\text { Security } \\
\text { evel }\end{array}$ & $\begin{array}{l}\text { Long-term } \\
\text { Security }\end{array}$ \\
\hline Fingerprint & Medium & Small & Low & Low & Low \\
\hline $\begin{array}{l}\text { Facial } \\
\text { Recognition }\end{array}$ & Low & Large & High & Low & Low \\
\hline Iris Scan & High & Large & High & Medium & Medium \\
\hline $\begin{array}{l}\text { Voice } \\
\text { Recognition }\end{array}$ & Low & Small & Medium & Low & Low \\
\hline $\begin{array}{l}\text { Hand } \\
\text { Geometry }\end{array}$ & Low & Large & High & Low & Low \\
\hline Finger vein & High & Medium & High & High & High \\
\hline
\end{tabular}

In order to solve the inefficient of existing hand-based biometric systems, researchers [12][13] into finger vein recognition comes to the limelight to the finger vein because it has been verified a finger contains vein patterns, which are the networks of the blood vessel under the skin that the distinction is unique and different from each other. Whereas each individual has unique vein pattern even twin is different in vein patterns. Furthermore, authentication is obtained from the finger veins of a living person that means impossible to detect the vein from a dead person, thereby making it difficult to steal or forge the finger vein pattern. That is very useful in personal verification. Hence, studies [14] have confirmed that finger vein based biometric system has advantages more than others hands-based biometric. Since, replicating the finger vein pattern is difficult as it is an internal feature. Hence the condition of the epidermis such as wetness, dryness, finger 
pollution, and aging are not affecting the result of vein detection. Additionally, it only requires the person to place their fingers over a reader contactless and that makes authentication process easy to use, according to these advantages the study focused on finger vein as a high accuracy feature. The rest of the paper is organized as follow: Section II defines related works regarding finger vein recognition techniques. Section III illustrates the proposed technique in detail. Section IV presents the experimental results and Section V states the conclusion of the work.

\section{LITERATURE SURVEY}

In this section, an extensive review on finger vein recognition has been reported in detail by highlighting their strength and weaknesses Recently, Liu et al. 2018[15] presented a novel finger vein recognition algorithm by employing the use of a secure biometric template scheme based on deep learning and random projections that is called FVR-DLRP. This makes the use of biometric templates to be more secure, keep the original information even the passwords cracked. However, Deep learning needs huge data for training and computationally complex. While Dong et al. 2015[16] presented in their research on finger vein feature extraction method, a Multi- Orientation Weighted Symmetric Local Graph Structure (MOW-SLGS), which assigns weight to each edge with respect to the positional relationship between the edge and the target pixel. Their research also covered the use of the Extreme Learning Machine (ELM) which was expected to train and classify the vein feature extracted by the MOWSLGS method. However, extreme learning machine has random weights in the input -hidden layer which causes nonstable performance unless an optimization of the weights is done.

Other authors Song et al. 2011[17] proposed method, which referred to as the mean curvature method. This method considers the vein pattern as a geometric shape and finds the negative mean curvatures. However, it only focuses on feature extraction. On the other hand, Wu \& Liu 2011[18] used of a principal component analysis (PCA) as well as a linear discriminant analysis (LDA) and applied them to the image pre-processing as dimension reduction and feature extraction. In terms of the pattern classification, their system used an SVM and adaptive Neuro-fuzzy inference system (ANFIS) in which, they employed PCA method to remove noise residing in the discarded dimensions and retained the main feature by LDA. Hence, using that features in pattern classification and identification. However, the fuzzy approach has a lot of heuristics, a database includes a few numbers of subjects and the execution time is too long.

Then, Madhusudhan et al. 2018[19] proposed an algorithm that initially captured the finger-vein image and pre-processed using Gaussian blur and morphological operations. Then extract the features like a number of corner points and their location. Then, in order to test an authentication, the feature of an individual was fetched from the database and compared against the extracted features. If the comparison satisfies the predefined threshold value, then the authentication is successful. However, the threshold is a parameter that has to be tuned. Houjun Huang et al. 2017[20] used Deep Vein for finger vein verification based on deep convolutional neural networks. However, deep learning needs huge data for training and computationally complex. The accuracy of this method depends on the size of the training set, where it increases if the training set is large.

Next, Beining Huang et al. 2010[21] proposed a wide line detector method for feature extraction which allows obtaining accurate width information from the extracted feature then, developed a new pattern normalization model based on the supposition that the finger cross-sectional are similar to the ellipse and the vein denoted is closed to the finger surface which can reduce the distortion caused by the difference of finger structure. After four years Raghavendra et al. 2014[10] designed a new device distinguished with a low cost and one camera followed by near-infrared light to obtain high quality images for both fingerprint and finger vein at the same time, then used both the maximum curvature method and spectral minutiae representation (SMR) for feature extraction from the region of interest (ROI) of the finger vein image. KhellatKihel et al. 2014[22] presented a finger vein recognition system that uses a Support Vector Machine (SVM) based on a supervised training algorithm. This system used two methods in the pre-processing stage. The first method, included a median filter, histogram equalization, and segmentation while the second method, included a 2D Gabor filter, in order to assess the efficiency of the experiment in terms of its recognition rate. The finger vein recognition resides in the layer of machine learning where each individual classifier is trained on one of the developed features in the literature. Lastly, it will be interesting to incorporate an addition type of features to observe an additional improvement in the overall classifier. The objective of this paper is to design a geometrical feature for finger vein-based identification system.

\section{Proposed TechniQue}

In this section, the proposed technique namely StraightLine Approximation and its detail steps are explained comprehensively. The block diagram of the development phases of finger vein recognition system is shown in Fig. 1. The methodology of a novel system for finger vein recognition will be provided. Initially, provided the preprocessing of the images, resize all the images in the dataset and localized the region of interest (ROI). Then, it will go through several approaches of vein pattern detection from the (ROI). Also, this section will explain the combination rules to aggregate the individual classifier. The detail description of a block diagram components is explained below:

\section{A. Pre-Processing Images}

While passing the infrared through the skin, the finger region appears brighter than the background of the captured images. Therefore, most of these images contain shaded regions and noise at both sides of the finger. As a result, the pre-processing is combined based on three steps:

Step 1. Resize the image: this step includes reducing the number of pixels of the finger image without affecting the needed information for the identification. Therefore, all images in the dataset 
are resized to $(128 \times 96$ pixel $)$ to increase the processing speed.

Step 2. Contrast adjustment is another important step, in order to highlight the vein data and separate it from the background, that gives a better result for all the following steps in the identification.

Step 3. The Region of Interest Detection or (ROI) detection: this step is important for reducing the computational complexity by selecting the subregion in the finger vein image in which the processing will be performed. This sub-region contains the needed information of the vein. For this purpose, the study adopts the Lee algorithm [23] to localizing (ROI) for normalization and feature extraction by using Mask filter. as shown in Fig. 2.

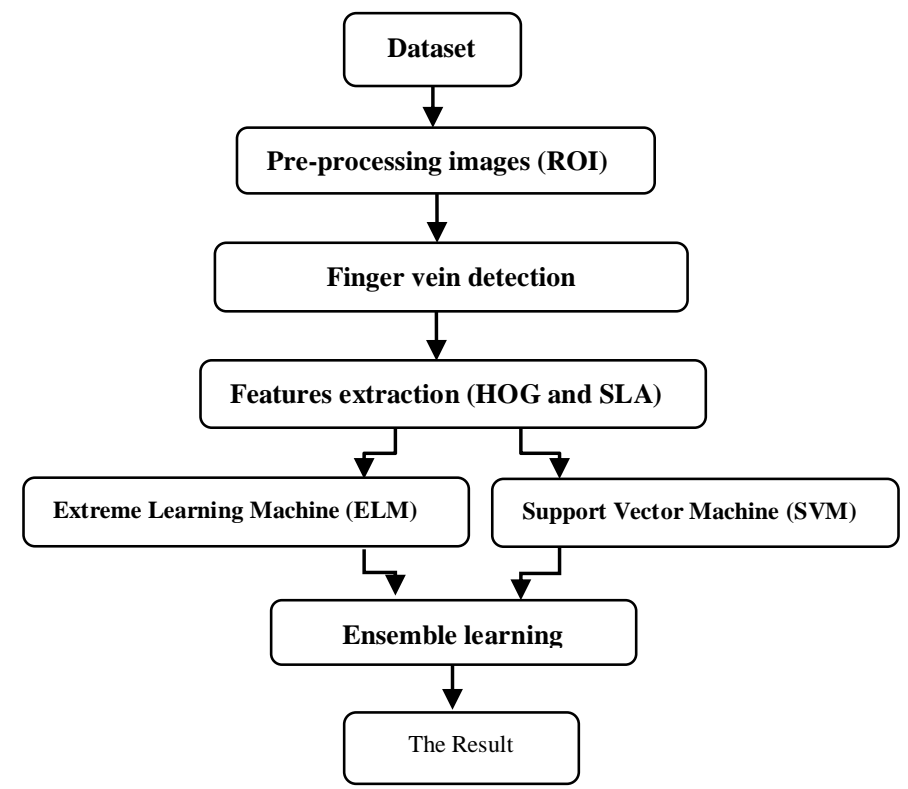

Fig 1. The Development Phases of Finger Vein Recognition System.

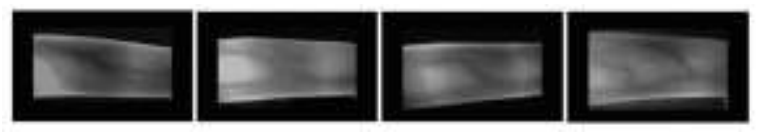

Fig 2. Localization of Different Fingers Region with the Mask.

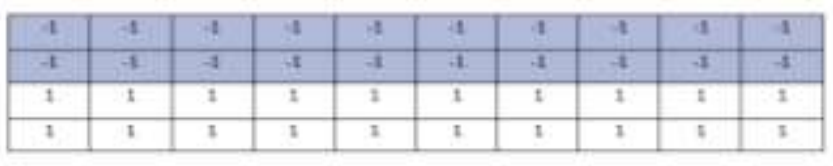

[A]

\begin{tabular}{|c|c|c|c|c|c|c|c|c|c|}
\hline 1 & $t$ & 1 & 1 & 3 & 1 & 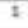 & I & $\mathrm{t}$ & 1 \\
\hline$t$ & 1 & 1 & 1 & 1 & 1 & 1 & 1 & 7 & 1 \\
\hline-1 & 1 & -4 & -1 & -4 & -1 & 4 & -2 & -4 & 4 \\
\hline 4 & $t$ & 7 & 4 & 13 & 1 & 4 & 4 & 4 & 4 \\
\hline
\end{tabular}

ins

Fig 3. Masks to Localize the Finger Region of the Image. (A) Detect the Upper Region of the Finger, (B) Detect the Lower Region of the Finger. Source [23].
The masking values are counted as shown in Fig. 3 (A and $\mathrm{B})$, from the $\mathrm{Y}$ direction and each $\mathrm{X}$ position, including the position at which the masking value becomes maximal, are considered by the boundary position between the background and finger in the Y direction [23].

\section{B. Finger Vein Detection}

The present study adopts three approaches to detect vein pattern from (ROI) extraction that has been proven providing good results through the previous literature. Those are; Maximum Curvature Method (MCM), Wide Line Detector (WLD), and Repeated Line tracking (RL) subsequently.

1) Maximum curvature method: This method used an algorithm to extract the vein pattern from finger images [24], that consists of three steps. The details are as follows:

\section{a) Extract the Center Position of the Veins}

Eventually, it produces the cross-sectional profile of the finger-vein in the image. In which the cross-sectional profile appears such as a ravine. Once the infrared passing through the finger, the vein objects appear darker than the background of the image. The position of the center lines of the veins is confirmed by assigning marks to each position, whereby the large mark is used as the deepest ravine of the vein in the finger image. This is followed by four stages: Firstly, calculation of the maximum curvatures in the cross-sectional profile to get the centerline position of the veins. Secondly, classifications were made to the curvatures of the crosssectional profile. Thirdly, assigning a score to each center position that represents the probability of the center positions are on the vein. Finally, in order to get the vein pattern pervasion in an image, the profiles of the vein in four directions are analyzed to get the vein pattern pervasion in all these directions, which they are vertical, horizontal and viz à viz the two-diagonal intersecting the vertical and horizontal directions at $45^{\circ}$. Thereby all the center positions are obtained by counting the maximum curvature.

\section{b) Connect the Center Position}

In order to connect the center points and remove noise, it is necessary to constantly check the two neighboring pixels on the right side and the left side of the pixel, especially if the value of the pixel and both right and left sides of the pixel are high, a line would be painted horizontal. On the other hand, if the value of the pixel is low and both sides of the pixel value are high, a line would be painted with a little gap at this pixel. As a result, it increases the pixel value to alleviate the gap and connect the line of the vein.

\section{c) Labelling the Images}

This step involves labeling the vein pattern by means of using a threshold, if the pixels with value are smaller than the threshold, label it as a part of the background. Else, if the pixels with value are bigger than the threshold, label it as a part of vein region. Here, the threshold is determined by maximizing the dispersion between the groups of values in the pixel.

2) Repeated line tracking: This method adopts the repeated tracking [25] on the dark lines of the vein pattern of the finger image purposely for obtaining a robust extraction 
feature. It uses any pixel of the image and considers the position of the pixel as a starting point $\left(x_{0}, y_{0}\right)$. Thereafter, moving pixel by pixel within the dark line of the vein. where, the depth of the cross-sectional profile of the vein appears as a ravine. Based on this, it is easy to detect the direction of the dark line by checking the depth of the cross-sectional profile (ravine) with the varying $\theta i$, and obtaining the deepest ravine subsequently. Later, the starting point track can be moved to the neighbor pixel along this direction.

Conversely, only a part of the veins within the image will be tracked if only single line-tracking operation is conducted. Therefore, to solve this issue, a vein-tracking should be started at various positions to determine the line-tracking trials which must be conducted equally across the image. This algorithm repeated about $n$ times to detect the dark line according to the following steps:

Step 4. Define the starting point for line tracking and the moving-direction attribute.

Step 5. Detect the direction of the dark line and the movement of the tracking point.

Step 6. Update the number of time points in the area space that have been tracked.

Step 7. Repeat implementing steps (1 and 3) with $n$ times.

Step 8. Gain the finger vein pattern from the area space.

3) Wide line detector: This method used to extract the vein pattern by disregarding the thickness of the line [21] based on, a hypothesis as shown in Fig. 4. It coherently considers the cross-sectional profile of the finger vein is approximately ellipses [26].

This algorithm describes 0 as the values of pixels in the feature image as parts of the background and 255 as the values of pixels as parts of the vein region. As a result, Fig. 4 shows $\left(x_{0}, y_{0}\right)$ denoted to a pixel at the center of the circle, while $(x, y)$ any other pixel within the circle, and $\mathrm{N}\left(x_{0}, y_{0}\right)$ is the brightness of the pixel.

\section{Feature Extraction}

Two main sets of features are used for this identification. The first one is the Histogram of Oriented Gradient (HOG) [27] features. HOG features were selected because of its robustness in the classification performance. The second one is a novel feature named Straight Line Approximation feature (SLA).

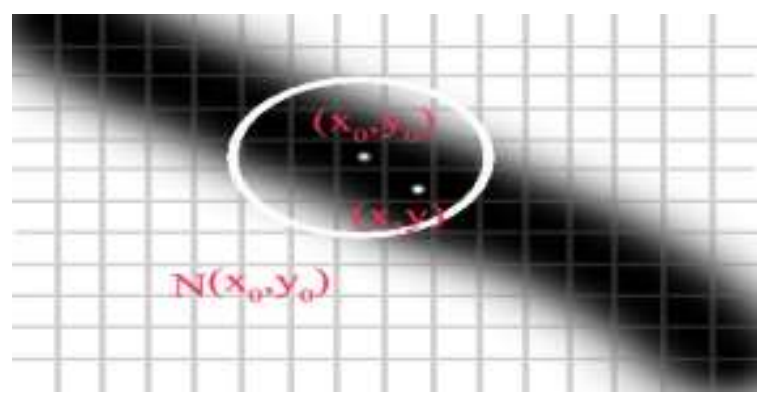

Fig 4. The Circular Vicinity Area.

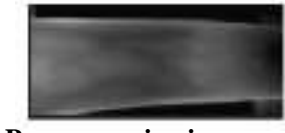

(a)
Pre-processing image

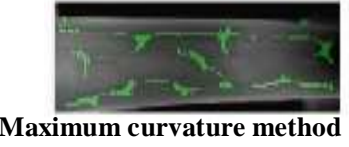

(b)

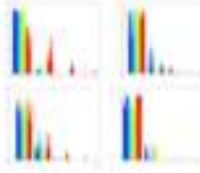

Histogram bin (d)

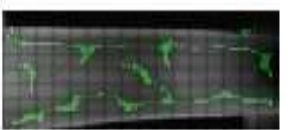

Histogram of Oriented Gradient

(c)
Fig 5. Histogram of Oriented Gradients Features.

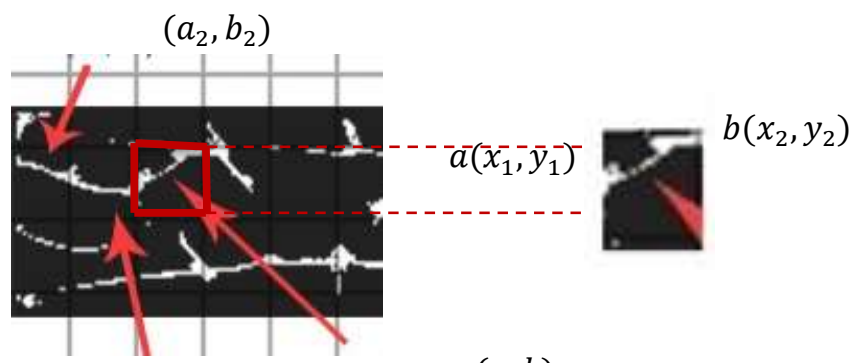

$(a, b)$

$\left(a_{1}, b_{1}\right)$

(a)

(b)

Fig 6. The Proposed Straight-Line Approximation Features.

The procedure of these features are including: Divide the pre-processing image into small sub-images $(8 \times 16)$ cells, each of these cells divided into blocks, the size of each block is $(1 \times 1)$ as shown in Fig. 5. Then, it accumulates a histogram of edge orientation by obtaining the vertical and horizontal gradients for each pixel within the cell. Next, it combines the histogram entries used as the feature vector describing the object. Since a gradient is affected by illumination changes, therefore it normalizes the cells across larger regions for overcoming illumination invariance. Hence, a histogram gradient descriptor is assigned to each block within the cell whereby it contains the information for recognizing the object. The study develops its own feature extraction method. In this approach, the finger vein image after pre-processing is divided into sub-blocks represented by a grid. It carries out a line fitting for the points for each sub-block. Each line combines two components namely a slop and offset. As the result, the aggregations of the components in all the sub-blocks are known as Straight-Line Approximation features or (SLA) as shown in Fig. 6.

The lines in the coordinate plane can be described by a linear equation with the two dimensions as shown in equation (1). The non-vertical lines are described in the slope-intercept form, as the equation (2):

$\mathrm{V}=\{(x, y) \mid a x+b y=c\}$,

$y=m x+b$, 
where $m$ represents a slope, $b$ is a line and $x$ is the separate variable of $y=f(x)$, the slope at the points $a\left(x_{1}, y_{1}\right) \cdot b\left(x_{2}, y_{2}\right)$ while $x_{1} \neq x_{2}$ is obtained by:

$m=\left(y_{2}-y_{1}\right) /\left(x_{2}-x_{1}\right)$

$y-y_{1}=\left(\left(y_{2}-y_{1}\right) /\left(x_{2}-x_{1}\right)\right)\left(x-x_{1}\right)$

$y=\left(\left(y_{2}-y_{1}\right) /\left(x_{2}-x_{1}\right)\right)\left(x-x_{1}\right)+y_{1}$

About two classifiers are deployed for testing and training the objects of (HOG and SLA) features namely Support Vector Machine and Extreme Learning Machine (ELM). MATLAB has been used for implementation.

\section{Classification}

There are two classifiers (ELM and SVM), in which each of them is trained on two types of features (HOG and SLA) separately. Also, each feature set is extracted from one of three detection approaches, such as a wide line detector, repeated line tracking and maximum curvature.

1) Support vector machines: Support Vector Machines (SVMs) are a set of related methods for supervised learning that is applicable to both regression and classification problems [28]. In this framework, learning means to estimate a function from a set of examples (the training sets). Therefore, in order to carry out its implementation, a learning machine must choose one function from a given set function, thereby minimizing a certain risk (the empirical risk). This means that the estimated function would be different from the actual (yet unknown) function. Additionally, the chosen set of functions as well as its training set, their risk depends on the complexity of the framework. Hence, a learning machine must find the best set of functions as determined by its complexity - and the best function in that set as represented in the following algorithm:

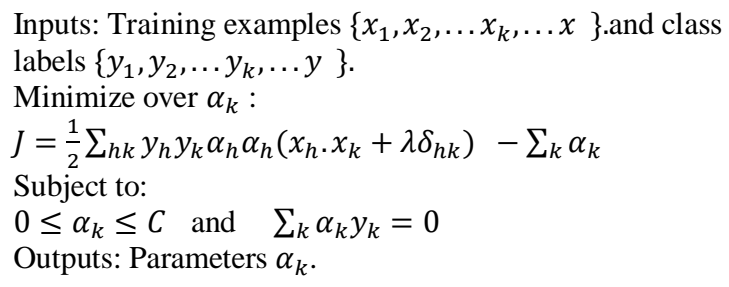

2) Extreme learning machine: This approach is about training one single hidden layer feed-forward neural network (SLFN). It is fast in performance because it uses small average weights that able to produce smaller training error in performance. Referring to the code presented by [29] the training combination is based on the following algorithm:

Step 1. Assign the weights for the input hidden layer were randomly

Step 2. Calculate the output hidden matrix

Step 3. Use Moore Penrose to find the hidden output matrix

\section{E. Ensemble Learning}

Ensemble learning method has enhanced the performance of multiple classifiers by aggregating the output of a set of classifiers. Each train applies one feature or more to produce an overall classification result [30].

1) Combination rules: The result of each classifier is presenting as a vector in the form of $\left(\alpha_{1} \alpha_{2} \ldots \alpha_{r}\right)$ where $r$ denotes to the number of classes. Each rule was applied on 12 classifiers. The classifiers are denoted as $E L M_{i . j}, S V M_{i . j}$ where $i=M C$. RL. WLD $j=H O G$.SLA as shown in Equation (6). The results of 12 classifiers are combining into single combination classifier using two rules [31] with the aim of enhancing the performance of the finger vein recognition system.

$y_{i s}=f\left(\left(\alpha_{1} \alpha_{2} \ldots \alpha_{r}\right)^{k}\right)=\left(y_{1} y_{2} \ldots y_{r}\right)$

We assumed $k$ is the number of the classifiers, $s$ is the output of each classifier for an entered image and $y_{i s}$ is the index of the outputs of the classifier.

a) The General Weighted of the Average Rule (GWAR)

Initially, this rule computes the global recognition rate of each classifier during the training step. Later, these values will be used to weight the average of the individual outputs, as shown in the Equation (7).

$y_{\text {ir }}=\frac{1}{k} \sum_{s=1}^{k} \mu_{s} \times y_{i s}, s=1, \ldots, k ; k=12$,

where $\mu_{s}$ the overall recognition-rate obtained by classifier during the training.

\section{b) The Dempster-Shafer (DS)}

This is an individual combination strategy. Based on decision templates, where $Z$ is the labeled training set, $Z=Z_{1}, \ldots, Z_{N}, Z_{j} \in R^{I},(N)$ is the number of elements, $(i)$ the number of features in the training set. A decision template $D T_{i}$ of class $i$ : and $n \times k$ matrix defined by Equation (8):

$D T_{i}(p, q)(Z)=\frac{\sum_{j=1}^{N} \operatorname{ind}\left(z_{j}, i\right) s_{p, q}\left(z_{j}\right)}{\sum_{j=1}^{N} \operatorname{ind}\left(z_{j}, i\right)}$

where $s$ is a matrix with two dimensions $p=1, \ldots, n$; $q=1, \ldots, k$ and $\operatorname{ind}\left(z_{j}, i\right)$ is an indicator function equal 1 if $z_{j}$ belongs to class $i$ and $=0$ otherwise. $D T_{i}(p, q)$ is the mean of the result obtained by the classifier $q$ to the all elements of class $q$ during the training step. The decision template $D T_{i}$ represents the average classifiers outputs of the training set $Z$ in class $i$. The decision profile of an entry $(x)$ is defined as the current template. Dempster-Shafer rule $(D S)$ use to an entry (x) to maximize the similarity between the decision template and decision profile. The $D S$ algorithm [32] assume $D T_{j}^{i}$ the row dimension $i=1, \ldots, n$ for the class $j=1, \ldots, k$ of the decision template, and $\Phi_{i, j}$ represents the approximate value between $D T_{j}^{i}$ and $D s(x)$ for every class $j=1, \ldots, k$ and any classifier $s=1, \ldots, n$. As given in equation (9).

$\phi_{j, i}(x)=\frac{\left(1+\left\|D T_{j}^{i}-D S(x)\right\|^{2}\right)^{-1}}{\sum_{P=1}^{k}\left(1+\left\|D T_{p}^{j}-D S(x)\right\|^{2}\right)^{-1}}$ 
where $\|$.$\| any matrix normal.$

Then, compute the belief degrees for every class $j=$ $1, \ldots, k$ and any classifier $s=1, \ldots, n$ as the following equations (10) and (11) subsequently:

$b_{j}(D S(x))=\frac{\phi_{j, s}(x) \Pi_{k \neq j}\left(1-\phi_{k, s}(x)\right)}{1-\phi_{j, s}\left[\prod_{k \neq j}\left(1-\phi_{k, S}(x)\right)\right]}$

$\mu_{\check{\mathrm{D}}}^{j}(x)=C \prod_{s=1}^{n} b_{j}(D S(x)), j=1, \ldots, k$,

where $C$ is a normalization constant which is empirically set as 0.5 .

\section{EXPERIMENTAL RESULTS}

In order to know which features are most effective for finger vein recognition, the use of SVM classifier is made with different kernels to compare the proposed feature with the work of [33] as the baseline work. The results are shown in Table 2.

We observed the maximum curvature method of vein detection has achieved the best results in terms of the accuracy in general for the two sets of features compared with the other two approaches of the finger vein detection. Also, observed this method achieved the best accuracy 0.8349 for (HOG) features. While the best result for (SLA) features 0.7783 using the polynomial and quadratic function.

Furthermore, Table 3 shows the classification results for different activation function of (ELM) using different neurons in the hidden layer with two types of feature extraction (HOG and SLA), and the three (ROI) detection algorithms.

TABLE II. NuMERICAL VALUES OF SVM ACCURACY, USING THREE APPROACHES (MAXIMUM CURVATURE, REPEATED LINE TRACKING AND

WIDE LINE DETECTOR) OF VEIN DETECTION WITH FOUR TYPE OF THE

KERNELS (LINEAR, POLYNOMIAL, QUADRATIC, RBF) AND TWO TYPES OF FEATURES EXTRACTIONS (HOG AND SLA)

\begin{tabular}{|l|l|l|}
\hline $\begin{array}{l}\text { Numerical values of SVM/ } \\
\text { features }\end{array}$ & $\begin{array}{l}\text { Accuracy } \\
\text { Of (HOG) }\end{array}$ & $\begin{array}{l}\text { Proposed accuracy } \\
\text { of (SLA) }\end{array}$ \\
\hline SVM Linear/ MCM & $\mathbf{0 . 8 3 0 2}$ & $\mathbf{0 . 7 7 3 6}$ \\
\hline SVM Linear/ RLT & 0.7453 & 0.6226 \\
\hline SVM Linear/ WLD & 0.8066 & 0.7358 \\
\hline SVM Polynomial/ MCM & $\mathbf{0 . 8 3 4 9}$ & $\mathbf{0 . 7 7 8 3}$ \\
\hline SVM Polynomial/ RLT & 0.7689 & 0.6415 \\
\hline SVM Polynomial/ WLD & 0.8255 & 0.7406 \\
\hline SVM quadratic/ MCM & $\mathbf{0 . 8 3 4 9}$ & $\mathbf{0 . 7 7 8 3}$ \\
\hline SVM quadratic/ RLT & 0.7689 & 0.6415 \\
\hline SVM quadratic/ WLD & 0.8255 & 0.7406 \\
\hline SVM RBF/MCM & 0.8160 & $\mathbf{0 . 7 5 4 7}$ \\
\hline SVM RBF/RLT & 0.7642 & 0.6368 \\
\hline SVM RBF/WLD & $\mathbf{0 . 8 2 0 7}$ & 0.7264 \\
\hline
\end{tabular}

www.ijacsa.thesai.org

TABLE III. NuMERICAL VALUES OF ELM ACCURACY USING THREE APPROACHES OF VEIN DETECTION (MCM, RL, WLD) AND TWO TYPES OF FEATURE EXTRACTIONS (HOG AND SLA) WITH DIFFERENT KERNELS (HARDLIM, RADIAL, SIGMOID, SINE, TRIANGLE) WITH 1000 NEURONS

\begin{tabular}{|l|l|l|}
\hline Numerical values of ELM/ features & $\begin{array}{l}\text { Accuracy } \\
\text { Of (HOG) }\end{array}$ & $\begin{array}{l}\text { Proposed } \\
\text { accuracy of } \\
\text { (SLA) }\end{array}$ \\
\hline ELM hardlim/1000 neurons/MCM & $\mathbf{0 . 7 4 5 3}$ & $\mathbf{0 . 6 6 9 8}$ \\
\hline ELM hardlim /1000 neurons/ RL & 0.4764 & 0.3538 \\
\hline ELM hardlim /1000 neurons/ WLD & 0.6509 & 0.5708 \\
\hline ELM radial/1000 neurons/ MCM & 0.6132 & $\mathbf{0 . 0 2 3 6}$ \\
\hline ELM radial/1000 neurons/ RL & 0.4623 & 0.0094 \\
\hline ELM radial/1000 neurons/ WLD & $\mathbf{0 . 6 1 7 9}$ & 0.0142 \\
\hline ELM sigmoid/1000 neurons/ MCM & $\mathbf{0 . 7 8 7 7}$ & $\mathbf{0 . 6 4 6 2}$ \\
\hline ELM sigmoid/1000 neurons/ RL & 0.6368 & 0.3538 \\
\hline ELM sigmoid/1000 neurons/ WLD & 0.7123 & 0.5613 \\
\hline ELM sine /1000 neurons/ MCM & 0.5425 & 0.0094 \\
\hline ELM sine / 1000 neurons/ RL & 0.6132 & 0.0094 \\
\hline ELM sine/ 1000 neurons/ WLD & $\mathbf{0 . 6 2 2 6}$ & $\mathbf{0 . 0 0 4 7}$ \\
\hline ELM triangle/1000 neurons/ MCM & $\mathbf{0 . 5 4 2 5}$ & $\mathbf{0 . 0 1 8 9}$ \\
\hline ELM triangle/1000 neurons/ RL & 0.3208 & 0.0094 \\
\hline ELM triangle/1000 neurons/ WLD & 0.4717 & 0.0047 \\
\hline
\end{tabular}

TABLE IV. OVERALL VALUES OF THE ACCURACIES OF THE TWO RULES FOR THE DIFFERENT COMBINATION OF FINGER VEIN DETECTION METHODS, CLASSIFIERS, AND FEATURES AT RANK 1 AND RANK 5

\begin{tabular}{|l|l|l|}
\hline Combination rules & Rank 1 & Rank 5 \\
\hline GWAR & 0.8774 & 0.9245 \\
\hline DS & 0.8774 & 0.9340 \\
\hline
\end{tabular}

We observed from the results. The maximum curvature method, in general, achieved the best results in an accuracy, in the two set of features (HOG and SLA) compared with the other two approaches. The accuracy of (HOG) features 0.7877 better than (SLA) using sigmoid function. Also, observed the maximum curvature method achieved best result for (SLA) features 0.6698 with activation function hardlim using 1000 neurons.

The aggregation result of the three (ROI) detection methods with (HOG and SLA) features and the two classifiers (SVM and ELM) are shown in Table 4, using two rules (DS and GWAR).

The overall performance of the system has been improved by using combination rules. Based on the recognition rate at two ranks generated, the obtained accuracy is approximately $87 \%$ for GWAR and DS at rank 1 while for rank 5, it is approximately $93 \%$ for DS and $92 \%$ for GWAR. 


\section{Conclusion}

The main problems at hand are how to solve the issues in human identity recognition and how to protect information security. We proposed to solve this issue by developing a new type of geometrical features that is called straight-line approximator (SLA) using ensemble learning. The comparison in this work was with state of the art approaches and more specifically on the work of [33] as a benchmark. Also, we used the combination rules based on 12 classifiers to improve the performance of the system. In this paper MATLAB environment version 2017a has been used because it is a wellknown development environment for image processing and machine learning. It has a rich library for a wide range of mathematical and artificial intelligence models including (SVM) functions. A public data set SDUMLA-HMT have been used which its collected by Joint Lab for Intelligent Computing and Intelligent Systems of Wuhan University [34]. The experiment result showed (DS and GWAR) rules have achieved an accuracy of $87 \%$ at rank 1, while the accuracy of DS rule $93 \%$ and GWAR rule $92 \%$ at rank 5 .

\section{ACKNOWLEDGMENT}

This research was funded by Ministry of Higher Education through UKM Research Fund AP2017-005/2.

\section{REFERENCES}

[1] E. Sundararajan et al., "USER AUTHENTICATION FOR ONLINE EXAMINATION BASED ON LOGIN, PREFERENCES AND MULTIMODAL-BIOMETRIC AUTHENTICATIONS."

[2] G. J. Westerhof, M. Maessen, R. De Bruijn, and B. Smets, "MultipleFrames Super-Resolution for Closed Circuit Television Forensics," Aging Ment. Heal., vol. 12, no. 3, pp. 317-322, 2008.

[3] A. J. Teoh and S. A. Samad, "DECISION FUSION COMPARISON FOR A BIOMETRIC VERIFICATION SYSTEM USING FACE AND," vol. 15, no. 2, pp. 17-27, 2002.

[4] S. Islam, M. S. Bhuyan, S. H. M. Ali, M. Othman, and B. Y. Majlis, "VHDL Implementation of Fuzzy Based Handwriting Recognition System," pp. 188-191, 2010.

[5] K. A. Rhodes, "Information Security: Challenges in Using Biometrics," Inf. Secur., 2003.

[6] A. N. Hoshyar1, R. Sulaiman2, and A. N. Houshyar, "SMART ACCESS CONTROL WITH FINGER VEIN AUTHENTICATION AND NEURAL," vol. 7, no. 9, pp. 192-200, 2011.

[7] S. Z. A. Rahman, S. N. H. S. Abdullah, and M. Z. B. A. Nazri, "The analysis for Gait Energy Image based on statistical methods," 2016 Int. Conf. Adv. Electr. Electron. Syst. Eng. ICAEES 2016, no. July 2018, pp. 125-128, 2017.

[8] Y. Zhou and A. Kumar, "Human identification using palm-vein images," IEEE Trans. Inf. Forensics Secur., vol. 6, no. 4, pp. 12591274, 2011.

[9] A. K. Jain, A. Ross, S. Pankanti, and S. Member, "Biometrics: A Tool for Information Security," vol. 1, no. 2, pp. 125-143, 2006.

[10] R. Raghavendra, K. B. Raja, J. Surbiryala, and C. Busch, "A low-cost multimodal biometric sensor to capture finger vein and fingerprint," IJCB 2014 - 2014 IEEE/IAPR Int. Jt. Conf. Biometrics, 2014.

[11] R. Narinder, "Comparison of Various Biometric Methods," Int. J. Eng. Sci. Technol., vol. 2, no. I, pp. 24-30, 2014.

[12] C. Qin, L. Shuncheng, Z. Huizhe, and Z. Jon, "Finger-vein Authentication Based on Wide Line Detector and Pattern Normalization Beining," Polyhedron, vol. 8, no. 24, pp. 2915-2923, 1989.

[13] D. Mulyono and H. S. Jinn, "A Study of Finger Vein Biometric for Personal Identification," 2008.

[14] A. N. Hoshyar, "Review on Finger Vein Authentication System by Applying Neural Network," pp. 1020-1023, 2010.
[15] Yi Liu, J. Ling, Z. Liu, J. Shen, and C. Gao, "Finger vein secure biometric template generation based on deep learning," Soft Comput., vol. 22, no. 7, pp. 2257-2265, 2018.

[16] S. Dong, J. Yang, C. Wang, Y. Chen, and D. Sun, "A New Finger Vein Recognition Method Based on the Difference Symmetric Local Graph Structure (DSLGS)," Int. J. Signal Process. Image Process. Pattern Recognit., vol. 8, no. 10, pp. 71-80, 2015.

[17] W. Song, T. Kim, H. C. Kim, J. H. Choi, H. J. Kong, and S. R. Lee, "A finger-vein verification system using mean curvature," Pattern Recognit. Lett., vol. 32, no. 11, pp. 1541-1547, 2011.

[18] J. Da Wu and C. T. Liu, "Finger-vein pattern identification using SVM and neural network technique," Expert Syst. Appl., vol. 38, no. 11, pp. 14284-14289, 2011.

[19] M. V Madhusudhan, R. Basavaraju, and C. Hegde, Secured Human Authentication Using Finger-Vein Patterns, vol. 839. Springer Singapore, 2018.

[20] Houjun Huang, S. Liu, H. Zheng, L. Ni, Yi Zhang, and W. Li, "DeepVein: Novel finger vein verification methods based on Deep Convolutional Neural Networks," 2017 IEEE Int. Conf. Identity, Secur. Behav. Anal., no. 5, pp. 1-8, 2017.

[21] Beining Huang, Y. Dai, R. Li, D. Tang, and W. Li, "Finger-vein authentication based on wide line detector and pattern normalization," Proc. - Int. Conf. Pattern Recognit., vol. 1, pp. 1269-1272, 2010.

[22] S. Khellat-Kihel, R. Abrishambaf, N. Cardoso, J. Monteiro, and M. Benyettou, "Finger vein recognition using Gabor filter and Support Vector Machine," Int. Image Process. Appl. Syst. Conf. IPAS 2014, pp. $1-6,2014$.

[23] Lee, H. C. Lee, and R. K. Park, "Finger Vein Recognition Using Minutia-Based Alignment and Local Binary Pattern-Based Feature Extraction Eui,” Int. J. Imaging Syst. Technol., vol. 19, no. 3, pp. 179186, 2009.

[24] N. Miura, A. Nagasaka, and T. Miyatake, "Extraction of finger-vein patterns using maximum curvature points in image profiles," IEICE Trans. Inf. Syst., vol. E90-D, no. 8, pp. 1185-1194, 2007.

[25] N. Miura, A. Nagasaka, and T. Miyatake, "Feature extraction of finger vein patterns based on iterative line tracking and its application to personal identification," Syst. Comput. Japan, vol. 35, no. 7, pp. 61-71, 2004.

[26] L. Liu, D. Zhang, and J. You, "Detecting wide lines using isotropic nonlinear filtering," IEEE Trans. Image Process., vol. 16, no. 6, pp. 1584-1595, 2007.

[27] S. Z. A. Rahman, S. N. H. S. Abdullah, and K. A. Z. Ariffin, "Gait Recognition based on Inverse Fast Fourier Transform Gaussian and Enhancement Histogram Oriented of Gradient," vol. 8, no. 4, pp. 1402 1410, 2018.

[28] G. Alipoor and E. Samadi, "Robust Speaker Gender Identification Using Empirical Mode Decomposition-Based Cepstral Features," vol. 7, no. 1, pp. 71-81, 2018.

[29] G. Huang, L. Chen, and C. Siew, "With Random Hidden Nodes," Ieee Trans. Neural Networks, vol. 17, no. 4, pp. 879-892, 2006.

[30] Y. Kessentini, T. Burger, and T. Paquet, "A Dempster-Shafer Theory based combination of handwriting recognition systems with multiple rejection strategies," Pattern Recognit., vol. 48, no. 2, pp. 534-544, 2015.

[31] Z. Tamen, H. Drias, and D. Boughaci, "An efficient multiple classifier system for Arabic handwritten words recognition," Pattern Recognit. Lett., vol. 93, pp. 123-132, 2017.

[32] G. Rogova, "Combining the results of several neural network classifiers,” Stud. Fuzziness Soft Comput., vol. 219, no. 5, pp. 683-692, 2008.

[33] M. A. Syarif, T. S. Ong, A. B. J. Teoh, and C. Tee, "Enhanced maximum curvature descriptors for finger vein verification," Multimed. Tools Appl., vol. 76, no. 5, pp. 6859-6887, 2016.

[34] Y. Yin, L. Liu, and X. Sun, "SDUMLA-HMT: A multimodal biometric database," Lect. Notes Comput. Sci. (including Subser. Lect. Notes Artif. Intell. Lect. Notes Bioinformatics), vol. 7098 LNCS, pp. 260-268, 2011. 